\title{
Ärzteschaft und HIN - wie weiter?
}

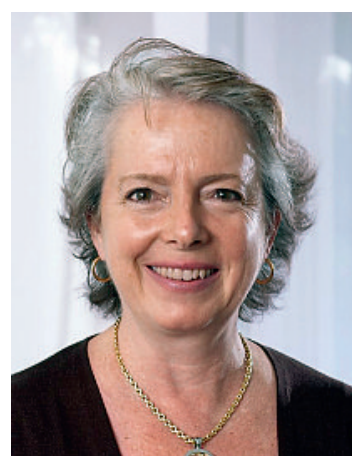

Im Mai 2009 hat die Ärztekammer HIN (Health Info Net) eine Überraschung bereitet: Sie hat es abgelehnt, dass die FMH auf ihre Stellung als Hauptaktionärin des Unternehmens verzichtet. Im Rahmen einer Kompromisslösung war schon alles vorbereitet, um die Aktien zu veräussern. Damit sollte auf die «Konkurrenzsituation» reagiert werden, die zwischen HIN und der HPC (Health Professional Card, dem Ausweis der Schweizer Ärzteschaft) zu bestehen schien und die zu Unstimmigkeiten und Unverständnis geführt hatten.

Dieser unerwartete Beschluss zwang uns zu intensiven Überlegungen: Wie lässt sich wieder eine Vertrauensbeziehung, eine fruchtbare Partnerschaft aufbauen, die für die Ärztinnen und Ärzte in Zukunft Lösungen bringt? Wir mussten gemeinsam abwägen, Ressentiments überwinden und danach wieder zusammen kreative Ansätze entwickeln.

\section{Die Übermittlung der medizinischen Daten über das Internet ist ein zentraler Aspekt für die Sicherheit der Daten unserer Patientinnen und Patienten}

In der Ära von Präsident H. H. Brunner hat die FMH die Entwicklung von zahlreichen Unternehmen unterstützt, die Lösungen für die Praxisadministration, Informatiktools oder die gesicherte Übermittlung und Verwaltung der Rechnungsdaten anbieten. Das geschah in den Anfangszeiten der Kosten- und Leistungsrechnung für die Spitäler, der Leistungskontrolle durch die Krankenkassen - ein gewaltiger Umbruch, der die Arbeit der Ärztinnen und Ärzte tiefgreifend veränderte. Die Lösungen zeigen die erstaunliche Innovationsfähigkeit der Verbindung der Schweizer Ärztinnen und Ärzte auf. In der Folge wurden diese Unternehmen selbständig. Die einzige Ausnahme schien HIN zu bilden, denn die
FMH blieb dort Hauptaktionärin. Der Entscheid, die Aktien wieder zu verkaufen, entsprach der allgemeinen Strategie der FMH, sich auf ihre Kernaufgabe zu konzentrieren: das Engagement für die politischen Aspekte und die Ausbildungsfragen im Zusammenhang mit dem Arztberuf.

\section{Die FMH hat den Verwaltungsrat von HIN mit Sicherheitsexperten verstärkt}

Andererseits ist die Übermittlung der medizinischen Daten über das Internet ein zentraler Aspekt für die Sicherheit der Daten unserer Patientinnen und Patienten und somit des Arztgeheimnisses, für das wir mit Überzeugung und Entschlossenheit eintreten. Somit war es gerechtfertigt, diese Übermittlung nicht aus der Hand zu geben - gerade in einer Zeit, in der die Vertraulichkeit der Daten immer wieder gefährdet ist. Denn mit den Schwachstellen im System wurde vielerorts Appetit geweckt, mit äusserst gravierenden Folgen für die Schweiz. Wir möchten verhindern, dass der Gesundheitssektor in eine ähnliche Lage gerät, wie sie zurzeit im Finanzbereich im Zusammenhang mit den gestohlenen Bankdaten zu beobachten ist, die sogar von staatlichen Stellen für teures Geld gekauft werden.

Dass die Ärztinnen und Ärzte in der Schweiz einen sicheren Ausweis wie die HPC benutzen, mit der sie ihre Qualifikation nachweisen können, und zusätzlich ihre Daten über ein geschlossenes, gesichertes Netzwerk wie HIN übermitteln, bietet Gewähr für das Label «Schweizer Qualität». Deshalb hat die FMH den Verwaltungsrat von HIN mit Sicherheitsexperten verstärkt, welche die Bereiche Identitätsnachweis SuisseID oder medizinische Informatik abdecken. Nun liegt es an uns allen, dieses «Netzwerk der Schweizer Ärzteschaft» aufzubauen, indem wir die HPC und HIN zusammen nutzen. Sicherheit hat zwar ihren Preis und ist mit gewissen Einschränkungen verbunden, doch ein Datenmissbrauch würde unendlich mehr kosten!

Monique Gauthey, Fachärztin FMH, Mitglied des Zentralvorstands der FMH, Verantwortliche für das Ressort eHealth 